\title{
Effects of 24 Weeks of Growth Hormone Treatment on Bone Microstructure and Volumetric Bone Density in Patients with Childhood-Onset Adult GH Deficiency
}

\author{
Hongbo Yang $\mathbb{D}^{1},{ }^{1}$ Kemin Yan, ${ }^{1}$ Yuping Xu, ${ }^{1}$ Linjie Wang ${ }^{\mathbb{D}},{ }^{1}$ Qi Zhang, ${ }^{2}$ Fengying Gong $\mathbb{D}^{1}$, \\ Huijuan Zhu $\mathbb{D}^{1},{ }^{1}$ Weibo Xia $\mathbb{D}^{1},{ }^{1}$ and Hui Pan $\mathbb{D}^{1}$ \\ ${ }^{1}$ Department of Endocrinology, Key Laboratory of Endocrinology of National Health Commission, \\ The Translational Medicine Center of PUMCH, Peking Union Medical College Hospital, \\ Chinese Academy of Medical Science and Peking Union Medical College, Beijing 100730, China \\ ${ }^{2}$ Department of Clinical Laboratory, Peking Union Medical College Hospital, \\ Chinese Academy of Medical Sciences and Peking Union Medical College, Beijing 100730, China
}

Correspondence should be addressed to Huijuan Zhu; shengxin2004@163.com,WeiboXia; xiaweibo8301@163.com, and Hui Pan; panhui20111111@163.com

Received 8 November 2019; Revised 4 January 2020; Accepted 17 January 2020; Published 13 March 2020

Academic Editor: Alberto Ferlin

Copyright (C) 2020 Hongbo Yang et al. This is an open access article distributed under the Creative Commons Attribution License, which permits unrestricted use, distribution, and reproduction in any medium, provided the original work is properly cited.

Objective. Adults with childhood-onset growth hormone deficiency (CO AGHD) have prominently impaired volumetric bone density (vBMD) and bone microarchitecture. Effects of recombinant human growth hormone (rhGH) on bone microarchitecture in CO AGHD were insufficiently evaluated. The objective of this study is to assess the effects of rhGH on bone microarchitecture and vBMD in CO AGHD patients. Design. In this single-center prospective study, nine CO AGHD patients received rhGH treatment for 24 weeks. High-resolution peripheral quantitative computerized tomography (HRpQCT) of distal tibia and radius was performed at baseline and at the end of treatment. Main outcomes were vBMD and morphometric parameters from HR-pQCT. Results. After 24-week treatment, IGF-1 SDS gradually increased from $-3.31 \pm 1.56$ to $-1.92 \pm 1.65(p=0.113)$. Serum phosphate $(1.17 \pm 0.17$ vs. $1.35 \pm 0.18 \mathrm{mmol} / \mathrm{L}, p=0.030)$, alkaline phosphatase $(83.6 \pm 38.6$ vs. $120.5 \pm 63.7, p=0.045)$, and $\beta$-CTX $(0.67 \pm 0.32$ vs. $1.09 \pm 0.58, p=0.022)$ were significantly elevated. In distal tibia, total vBMD $\left(200.2 \pm 41.7\right.$ vs $\left.210.3 \pm 40.9 \mathrm{mg} \mathrm{HA} / \mathrm{cm}^{3}, p=0.017\right)$, cortical area $\left(89.9 \pm 17.7 \mathrm{vs} 95.5 \pm 19.9 \mathrm{~mm}{ }^{2}\right.$, $p=0.032)$, and cortical thickness $(0.891 \pm 0.197$ vs $0.944 \pm 0.239 \mathrm{~mm}, p=0.028)$ were significantly improved. Trabecular area decreased from $795.3 \pm 280.9$ to $789.6 \pm 211.4 \mathrm{~mm}^{2}(p=0.029)$. Trabecular bone volume fraction increased from $0.193 \pm 0.038$ to $0.198 \pm 0.036(p=0.027)$. In radius, cortical perimeter $(74.1 \pm 10.0$ vs $75.0 \pm 10.9 \mathrm{~mm}, p=0.034)$, trabecular thickness $(0.208 \pm 0.013$ vs $0.212 \pm 0.013 \mathrm{~mm}, p=0.008)$, trabecular separation $(0.743 \pm 0.175 \mathrm{vs} 0.796 \pm 0.199 \mathrm{~mm}$, $p=0.019)$, and inhomogeneity of network (Tb.1/N.SD) $(0.292 \pm 0.087$ vs $0.317 \pm 0.096 \mathrm{~mm}, p=0.026)$ were significantly improved, while trabecular number $(1.363 \pm 0.294$ vs $1.291 \pm 0.3251 / \mathrm{mm}, p=0.025)$ decreased significantly. Conclusions. Our results provide evidence for improvement of vBMD and bone microarchitecture in AGHD patients at a relatively early stage of rhGH treatment.

\section{Introduction}

Adult growth hormone deficiency (AGHD) is a debilitating condition associated with hereditary or acquired pituitary disorders. Main clinical characteristics include low bone mineral density, sarcopenia, increased risk of metabolic syndrome, and decreased quality of life $[1,2]$. Accumulating data suggested that low bone mass and increased fracture frequencies are important manifestations of AGHD [3-5]. AGHD can be divided into childhood-onset (CO AGHD) and adulthood-onset (AO AGHD). Most cases of GHD in children are idiopathic and transient, while GHD is usually 
permanent in multiple pituitary hormone deficiency (MPHD) [6]. The effects of $\mathrm{rhGH}$ replacement therapy in AGHD patients on various outcomes had been reported, and the data showed inconclusive results so far, one reason of which was selection bias especially with a mixture of $\mathrm{CO}$ AGHD and AO AGHD patients [7].

rhGH promotes linear growth and healthy development in childhood and plays crucial roles in normal body function in adulthood, including maintenance of bone strength, muscle volume, and other metabolic parameters [1]. There are several clinical studies evaluating the effects of $\mathrm{rhGH}$ on bone mineral density. In patients with both AO AGHD and $\mathrm{CO} A \mathrm{AHD}, \mathrm{rhGH}$ replacement therapy was reported to have various beneficial effects on bone mineral density (BMD) and total bone mineral content (BMC) [8-11]. Bone biopsy showed increased osteoid surface, osteoid volume, and activation frequency, but trabecular bone volume did not increase significantly [11]. In these studies, patients received 18 months to five years of $\mathrm{rhGH}$ treatment, and assessment of bone mineral density and bone mineral content were based on dual-energy X-ray absorptiometry (DXA) at the hip and lumbar spine. DXA evaluates 2D areal bone mineral density (aBMD) and provides no information of microarchitecture of cortical and cancellous bones. Thus, it will take a relatively long term of treatment to assess aBMD changes in AGHD patients by DXA. High-resolution peripheral quantitative computerized tomography (HR-pQCT) provides details of bone microarchitecture and volumetric bone mineral density (vBMD) $[12,13]$. In our previous report, we found that young adults with childhood-onset growth hormone deficiency (CO AGHD) have prominently impaired vBMD and bone microarchitecture [14]. In the present study, effects of 24-week rhGH replacement therapy on bone microstructure and vBMD in male patients with $\mathrm{CO}$ AGHD were evaluated.

\section{Subjects and Methods}

2.1. Patients. A total of nine consecutive male patients with CO AGHD were enrolled in this prospective study from April 2017 to January 2019 in our GHD clinic at Peking Union Medical College Hospital [15]. The diagnosis of AGHD was made according to the criteria of American Endocrine Society Clinical Practice Guideline [1]. All patients had childhood-onset multiple pituitary hormone deficiency. Eight patients stopped rhGH replacement therapy since the attainment of final height. One patient did not receive rhGH treatment before. All patients sustained glucocorticoid and levothyroxine replacement since childhood. Thyroid function test was monitored to titrate $\mathrm{LT}_{4}$ dosage. Body weight, blood pressure, and electrolytes were monitored routinely to avoid overdose of glucocorticoids, since both overtreatment of glucocorticoids and levothyroxine can impair bone framework [16]. Oral testosterone replacement was started after completion of linear growth, and testosterone undecanoate intramuscular injection monthly was maintained in the past five years. AGHD was diagnosed with peak GH value lower than $3 \mu \mathrm{g} / \mathrm{L}$ in insulin tolerance test.
2.2. Study Protocol. During 24-week treatment, the daily rhGH dose was started from subcutaneous injection of 1.0 IU per night and titrated in the event of side-effects and on an individual basis if serum IGF-1 concentration was higher than the age- and gender-adjusted reference values [1].

All patients were followed at our GHD clinic. At the start of the study and every eight weeks, physical and laboratory examinations were performed. Anthropometric parameters including height, weight, waist circumference, and hip circumference were measured with standard protocols in early morning with light clothes. BMI was calculated as weight $(\mathrm{kg})$ divided by height $(\mathrm{m})$ squared. Bioelectric impedance assessments (BIA) by the body composition analyzer (Tanita TBF-410, Japan) were performed in the morning after overnight fasting according to manufacturer's instructions. Fat-free mass (FFM), fat-free mass percentage (FFM\%), fat mass (FM), FM percentage (FM\%), and total body water (TBW) were obtained according to the body composition model. FFM index and FM index were calculated as described previously [17]. Grip strength was measured with a hand dynamometer (Jamar Plus+, Sammons Preston, Rolyon, Bolingbrook, IL, USA) on the nondominant hand, as described previously [14].

Informed consent was obtained from all patients. The study was approved by the Institutional Review Board of Peking Union Medical College Hospital (Beijing, China). All data were de-identified before analysis.

2.3. Biochemical Measurements. Blood samples were obtained in the morning after an overnight fasting. The serum IGF-1 level was measured with a fully automated two-site, solid-phase, chemiluminescent enzyme immunometric assay (Immulite 2000 ${ }^{\circledR}$, Siemens Healthcare Diagnostics, UK). Liver function, kidney function, $\mathrm{HbA1c}$, serum calcium, serum phosphate, alkaline phosphatase (ALP), 25-hydroxyvitamin D (25 OHD), parathyroid hormone (PTH), $\beta-\mathrm{C}-$ terminal telopeptide region of collagen type 1 ( $\beta$-CTX), and hormonal evaluation were all tested in the department of clinical laboratory of Peking Union Medical College Hospital by standard methods.

2.4. Volumetric Bone Density and Microarchitecture Assessment by HR-pQCT. High-resolution images of the nondominant distal radius and distal tibia were obtained by HRpQCT scan (XtremeCT II scanner, ScancoMedical, Brüttisellen, Switzerland) as reported previously [14, 18]. Briefly, when a scout scan was finished, reference lines were placed at the distal end plates for both the radius and tibia. Each scan was comprised of 168 slices, corresponding to a $10.2 \mathrm{~mm}$ scan area, with a nominal isotropic resolution of $61 \mu \mathrm{m}$ carried out at the standard location $9.0 \mathrm{~mm}$ (radius) and $22.0 \mathrm{~mm}$ (tibia) proximal to the reference line. All scans were finished by a specified technologist and analyzed according to the standard manufacturer's method. Scans were graded for motion artifacts as described previously [19]. 
Bone microarchitecture parameters were obtained with the standard morphologic analysis by semiautomated software, including trabecular area (Tb.Ar), cortical area (Ct.Ar), cortical perimeter (Ct.Pm), total volume bone mineral density (Tt.vBMD), trabecular vBMD (Tb.vBMD), cortical vBMD (Ct.vBMD), trabecular separation (Tb.Sp), in-homogeneity of network (Tb.1/N.SD), cortical thickness (Ct.Th), and cortical porosity (Ct.Po) [20].

2.5. Statistical Analysis. Data with normal distribution are presented as mean \pm standard deviation (SD), and the paired-samples $t$ test was used for data analysis. Non-normal data are presented as median and ranges, and Wilcoxon signed-rank test was used for data analysis. All of the statistical computations were run using SPSS software version 22.0 for Windows (SPSS Inc., Chicago, IL, USA), and $p<0.05$ was considered to be statistically significant.

\section{Results}

3.1. Demographic, Clinical, and Biochemical Characteristics. General characteristics of CO AGHD patients at baseline and after 24-week rhGH replacement therapy are shown in Table 1. A total of 9 male patients with CO AGHD with a mean age of 28 years (range, 21-51) were enrolled in this prospective study. Five patients had pituitary stalk interruption, and four patients had pituitary hypoplasia based on MRI. Eight patients stopped rhGH replacement after completion of linear growth. Duration of rhGH replacement treatment was $11.5 \pm 3.1$ years. The average time course since the cessation of rhGH treatment was $7.1 \pm 3.3$ years. One patient never accepted rhGH treatment before, and he was 51 years old at the time of the start of this study. All patients had multiple pituitary hormone deficiency and sustained glucocorticoid and levothyroxine replacement since childhood. During the 24-week study, all patients accepted $250 \mathrm{mg}$ of testosterone undecanoate intramuscular injection monthly. All patients had no signs of diabetes insipidus. No clinical fracture was reported from all our patients.

After 24 weeks of treatment, there was a 0.83 increase in BMI $(p=0.014)$. No significant changes were found in waist-hip ratio. Fat mass percentage decreased from $31.6 \%$ to $29.1 \%(p=0.043)$. Fat mass index decreased from 7.20 to $6.20(p=0.012)$. No significant changes were found in fatfree mass index. Grip strength of nondominant hand had not been improved at the end of study (Table 1).

Endocrinological and biochemical parameters are listed in Table 2. After 24 weeks of rhGH replacement therapy, serum IGF-1 levels tended to increase from $74.8 \mathrm{ng} / \mathrm{ml}$ to $125.6 \mathrm{ng} / \mathrm{ml} \quad(p=0.090)$. IGF-1 SDS increased from $-3.31 \pm 1.56$ to $-1.92 \pm 1.65(p=0.113)$. With constant replacement of levothyroxine, FT3 was stable during the study and FT4 levels decreased from $1.04 \mathrm{ng} / \mathrm{dl}$ to $0.81 \mathrm{ng} / \mathrm{dl}$ $(p=0.024)$.

No routine calcium or vitamin D supplementation was prescribed during the study. Serum calcium levels remained stable after 24 weeks treatment. There were significant increases in serum phosphate $(1.17 \pm 0.17$ vs. $1.35 \pm 0.18 \mathrm{mmol} /$
$\mathrm{L}, p=0.030)$, serum alkaline phosphatase levels $(83.6 \pm 38.6$ vs. $120.5 \pm 63.7, p=0.045)$, and $\beta$-CTX $(0.67 \pm 0.32$ vs. $1.09 \pm 0.58, p=0.022)$ after 24 weeks of treatment. Serum $25 \mathrm{OHD}$ decreased from $22.05 \mathrm{ng} / \mathrm{ml}$ to $17.78 \mathrm{ng} / \mathrm{ml}$ $(p=0.032)$.

There was no difference in other biochemical parameters including fasting glucose, hemoglobulin, ALT, AST, albumin and uric acid at baseline and after 24 weeks of treatment.

3.2. Effects of 24-Week rhGH Treatment on $v B M D$ and Microarchitecture of the Distal Tibia. Data obtained by HRpQCT of the distal tibia are listed in Table 3. After 24week rhGH replacement therapy, there were significant improvements of total vBMD $(200.2 \pm 41.7$ vs $210.3 \pm$ $\left.40.9 \mathrm{mg} \cdot \mathrm{HA} / \mathrm{cm}^{3}, p=0.017\right)$, trabecular bone volume fraction $(0.193 \pm 0.038$ vs. $0.198 \pm 0.036, p=0.027)$, cortical area $\left(89.9 \pm 17.7\right.$ vs $\left.95.5 \pm 19.9 \mathrm{~mm}^{2}, p=0.032\right)$, and cortical thickness $(0.891 \pm 0.197$ vs $0.944 \pm 0.239 \mathrm{~mm}, p=0.028)$. There was significant decrease in trabecular area $(795.3 \pm$ 280.9 vs. $\left.789.6 \pm 211.4 \mathrm{~mm}^{2}, p=0.029\right)$. No significant changes were found in cortical vBMD, cortical perimeter, and intracortical porosity of the distal tibia. No significant changes were found in trabecular vBMD, trabecular thickness, and trabecular number or trabecular separation after 24 weeks of rhGH treatment. The detailed changes of some important HR-pQCT parameters of the distal tibia at baseline and after 24 weeks of rhGH treatment are presented in Figure 1.

3.3. Effects of 24-Week rhGH Treatment on $v B M D$ and Microarchitecture of the Distal Radius. Data obtained by HRpQCT of the distal radius are listed in Table 4. After 24-week rhGH replacement therapy, there were significant improvements of cortical perimeter $(74.1 \pm 10.0$ vs $75.0 \pm 10.9 \mathrm{~mm}, \quad p=0.034)$, trabecular thickness $(0.208$ \pm 0.013 vs $0.212 \pm 0.013 \mathrm{~mm}, p=0.008)$, and Tb.1/N.SD $(0.292 \pm 0.087$ vs $0.317 \pm 0.096 \mathrm{~mm}, p=0.026)$. There was a significant decrease in trabecular number $(1.363 \pm 0.294$ vs $1.291 \pm 0.3251 / \mathrm{mm}, p=0.025)$ and increase in trabecular separation $(0.743 \pm 0.175$ vs $0.796 \pm 0.199 \mathrm{~mm}, p=0.019)$. No significant changes were found in total vBMD, cortical area, cortical thickness, and trabecular vBMD or trabecular area after 24 weeks of rhGH treatment. The detailed changes of some important HR-pQCT parameters of the distal radius at baseline and after 24 weeks of rhGH treatment are presented in Figure 2.

\section{Discussion}

In our previous work, we found that adult male patients with $\mathrm{CO}$ AGHD who are no longer receiving GH replacement have abnormalities in bone microarchitecture and estimated bone strength [14]. In this pilot study, we further investigated the effects of short term rhGH replacement on bone microarchitecture in $\mathrm{CO}$ AGHD patients. Our results showed that rhGH significantly improved vBMD in distal tibia and bone microarchitecture in both distal tibia and 
TABLE 1: Anthropometric characteristics of nine patients at baseline and after 24 weeks' treatment.

\begin{tabular}{|c|c|c|c|c|}
\hline & Baseline & 24-week follow-up & Changes & $p$ value \\
\hline Height $(\mathrm{cm})$ & $161.9 \pm 9.0$ & $162.6 \pm 9.2$ & $0.00(0,2.00)$ & 0.050 \\
\hline Weight (kg) & $68.5 \pm 15.8$ & $71.5 \pm 17.3$ & $2.92 \pm 2.52$ & 0.008 \\
\hline Waist circumference $(\mathrm{cm})$ & $95.5 \pm 11.0$ & $94.0 \pm 12.4$ & $-1.49 \pm 2.90$ & 0.162 \\
\hline Hip circumference $(\mathrm{cm})$ & $97.9 \pm 10.5$ & $98.4 \pm 11.2$ & $2.00(-9.00,4.00)$ & 0.711 \\
\hline Waist-hip ratio & $0.97 \pm 0.04$ & $0.95 \pm 0.06$ & $-0.19 \pm 0.48$ & 0.272 \\
\hline $\mathrm{FM}(\mathrm{kg})$ & $18.8 \pm 10.3$ & $16.3 \pm 9.8$ & $-2.44 \pm 1.29$ & 0.013 \\
\hline FM (\%) & $31.6 \pm 10.0$ & $29.1 \pm 10.0$ & $-4.72 \pm 2.83$ & 0.043 \\
\hline FFM (kg) & $42.7 \pm 8.8$ & $45.4 \pm 8.8$ & $4.28 \pm 4.00$ & 0.156 \\
\hline TBW (kg) & $31.3 \pm 6.4$ & $33.2 \pm 6.4$ & $3.14 \pm 2.95$ & 0.160 \\
\hline BMI $\left(\mathrm{kg} / \mathrm{m}^{2}\right)$ & $25.8 \pm 4.4$ & $26.6 \pm 4.9$ & $1.30(-0.80,1.50)$ & 0.014 \\
\hline FMI $\left(\mathrm{kg} / \mathrm{m}^{2}\right)$ & $7.2 \pm 3.3$ & $6.2 \pm 3.2$ & $-0.10 \pm 0.51$ & 0.012 \\
\hline FFMI $\left(\mathrm{kg} / \mathrm{m}^{2}\right)$ & $17.3 \pm 2.0$ & $18.8 \pm 2.6$ & $1.50 \pm 1.35$ & 0.068 \\
\hline Grip strength of nondominant hand $(\mathrm{kg})$ & $31.9 \pm 12.2$ & $29.1 \pm 14.4$ & $0.84 \pm 6.90$ & 0.757 \\
\hline
\end{tabular}

Data are presented as mean \pm SD or median and ranges. FM, fat mass; FFM, fat-free mass; TBW, total body water; BMI, body mass index; FMI, fat mass index; FFMI, fat-free mass index.

TABle 2: Baseline and follow-up of endocrinological and biochemical parameters.

\begin{tabular}{lccc}
\hline & Baseline & 24-week follow-up & Changes \\
\hline IGF-1 $(\mathrm{ng} / \mathrm{ml})$ & $78.1 \pm 59.3$ & $125.6 \pm 75.6$ & $47.43 \pm 62.04$ \\
IGF-1 SDS & $-3.31 \pm 1.56$ & $-1.92 \pm 1.65$ & $1.38 \pm 1.97$ \\
FT3 $(\mathrm{pg} / \mathrm{mL})$ & $2.81 \pm 0.61$ & $2.86 \pm 0.85$ & $0.04 \pm 0.75$ \\
FT4 $(\mathrm{ng} / \mathrm{dL})$ & $1.04 \pm 0.18$ & $0.81 \pm 0.26$ & $-0.23 \pm 0.21$ \\
Glu $(\mathrm{mmol} / \mathrm{L})$ & $4.98 \pm 0.62$ & $4.73 \pm 0.75$ & $-0.24 \pm 0.59$ \\
Hb $(\mathrm{g} / \mathrm{L})$ & $151.9 \pm 7.9$ & $148.0 \pm 9.0$ & $-3.89 \pm 8.25$ \\
ALT $(\mathrm{U} / \mathrm{L})$ & $18.6 \pm 7.2$ & $19.1 \pm 11.1$ & 0.880 \\
AST $(\mathrm{U} / \mathrm{L})$ & $24.0 \pm 4.2$ & $24.9 \pm 6.0$ & $-3.00(-8.00,32.00)$ \\
GGT $(\mathrm{U} / \mathrm{L})$ & $19.5 \pm 5.1$ & $16.1 \pm 3.9$ & $-0.50(-4.00,15.00)$ \\
ALP $(\mathrm{U} / \mathrm{L})$ & $83.6 \pm 38.6$ & $120.5 \pm 63.7$ & $-3.38 \pm 4.44$ \\
Alb $(\mathrm{g} / \mathrm{L})$ & $47.6 \pm 3.6$ & $47.4 \pm 2.8$ & $36.88 \pm 42.80$ \\
PA $(\mathrm{mg} / \mathrm{L})$ & $263.0 \pm 39.5$ & $259.5 \pm 36.5$ & $0.00(-6.00,2.00)$ \\
Tbil $(\mu \mathrm{mol} / \mathrm{L})$ & $9.9 \pm 3.6$ & $13.0 \pm 3.7$ & $-3.50 \pm 22.90$ \\
Dbil $(\mu \mathrm{mol} / \mathrm{L})$ & $3.5 \pm 1.4$ & $4.6 \pm 1.6$ & $3.02 \pm 3.09$ \\
Ca $(\mathrm{mmol} / \mathrm{L})$ & $2.37 \pm 0.08$ & $2.41 \pm 0.13$ & $1.13 \pm 0.88$ \\
P $(\mathrm{mmol} / \mathrm{L})$ & $1.17 \pm 0.17$ & $1.35 \pm 0.18$ & $0.04 \pm 0.11$ \\
25OHD $(\mathrm{ng} / \mathrm{ml})$ & $22.05 \pm 8.56$ & $17.78 \pm 7.72$ & $0.18 \pm 0.19$ \\
PTH $(\mathrm{pg} / \mathrm{ml})$ & $51.45 \pm 19.72$ & $45.12 \pm 26.22$ & $-4.28 \pm 5.34$ \\
$\beta$-CTX $(\mathrm{ng} / \mathrm{ml})$ & $0.67 \pm 0.32$ & $1.09 \pm 0.58$ & $-6.33 \pm 37.24$ \\
Cr $(\mu \mathrm{mol} / \mathrm{L})$ & $74.1 \pm 8.3$ & $77.1 \pm 8.7$ & $0.42 \pm 0.48$ \\
UA $(\mu \mathrm{mol} / \mathrm{L})$ & $438.5 \pm 78.6$ & $404.4 \pm 71.1$ & $3.00 \pm 7.50$ \\
\hline
\end{tabular}

Data are presented as mean \pm SD or median and ranges. IGF-1, insulin like growth factor 1; FT3, free triiodothyronine; FT4, free thyroxine; Glu, glucose; Hb, hemoglobin; ALT, alanine transaminase; AST, aspartate aminotransferase; GGT, gamma-glutamyltransferase; ALP, alkaline phosphatase; Alb, albumin; PA, prealbumin; Tbil, total bilirubin; Dbil, direct bilirubin; Ca, calcium; P, phosphate; $25 \mathrm{OHD}, 25$ hydroxy-vitamin D; PTH, parathyroid hormone; $\beta$-CTX, $\beta$-Cterminal telopeptide region of collagen type 1; Cr, creatinine; UA, uric acid.

TABLE 3: Baseline and follow-up HR-pQCT parameters of the distal tibia.

\begin{tabular}{|c|c|c|c|c|}
\hline & Baseline & 24-week follow-up & Changes & $p$ value \\
\hline Total vBMD $\left(\mathrm{mg} \mathrm{HA} / \mathrm{cm}^{3}\right)$ & $200.2 \pm 41.7$ & $210.3 \pm 40.9$ & $10.01 \pm 10.11$ & 0.017 \\
\hline Cortical area $\left(\mathrm{mm}^{2}\right)$ & $89.9 \pm 17.7$ & $95.5 \pm 19.9$ & $5.60 \pm 6.47$ & 0.032 \\
\hline Cortical vBMD $\left(\mathrm{mg} \mathrm{HA} / \mathrm{cm}^{3}\right)$ & $870.3 \pm 53.2$ & $857.4 \pm 60.0$ & $8.60(-78.60,21.00)$ & 0.311 \\
\hline Cortical perimeter $(\mathrm{mm})$ & $114.0 \pm 14.3$ & $114.4 \pm 14.2$ & $0.33 \pm 0.64$ & 0.157 \\
\hline Cortical thickness (mm) & $0.891 \pm 0.197$ & $0.944 \pm 0.239$ & $0.05 \pm 0.07$ & 0.028 \\
\hline Intracortical porosity & $0.008 \pm 0.003$ & $0.010 \pm 0.005$ & $0.001(-0.001,0.009)$ & 0.094 \\
\hline Trabecular area $\left(\mathrm{mm}^{2}\right)$ & $795.3 \pm 280.9$ & $789.6 \pm 211.4$ & $-5.71 \pm 6.44$ & 0.029 \\
\hline Trabecular vBMD $\left(\mathrm{mg} \mathrm{HA} / \mathrm{cm}^{3}\right)$ & $121.6 \pm 29.4$ & $128.0 \pm 23.0$ & $6.37 \pm 10.20$ & 0.098 \\
\hline Trabecular thickness (mm) & $0.245 \pm 0.034$ & $0.248 \pm 0.033$ & $0.003 \pm 0.011$ & 0.482 \\
\hline Trabecular number $(1 / \mathrm{mm})$ & $1.203 \pm 0.318$ & $1.253 \pm 0.311$ & $0.012(-0.020,0.219)$ & 0.102 \\
\hline Trabecular separation (mm) & $0.878 \pm 0.295$ & $0.841 \pm 0.269$ & $-0.004(-0.250,0.014)$ & 0.227 \\
\hline Tb.1/N.SD (mm) & $0.396(0.199,0.964)$ & $0.389(0.193,0.995)$ & $0.001(-0.108,0.031)$ & 0.597 \\
\hline Trabecular bone volume fraction & $0.193 \pm 0.038$ & $0.198 \pm 0.036$ & $0.004 \pm 0.005$ & 0.027 \\
\hline
\end{tabular}

Data are presented as mean \pm SD or median and ranges. vBMD, volumetric bone mineral density; Tb.1/N.SD, Std. dev. of 1/Tb.N, inhomogeneity of network. 


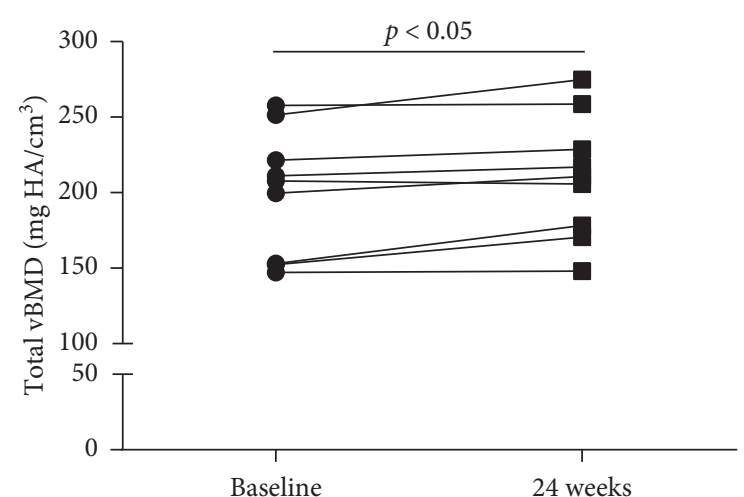

(a)

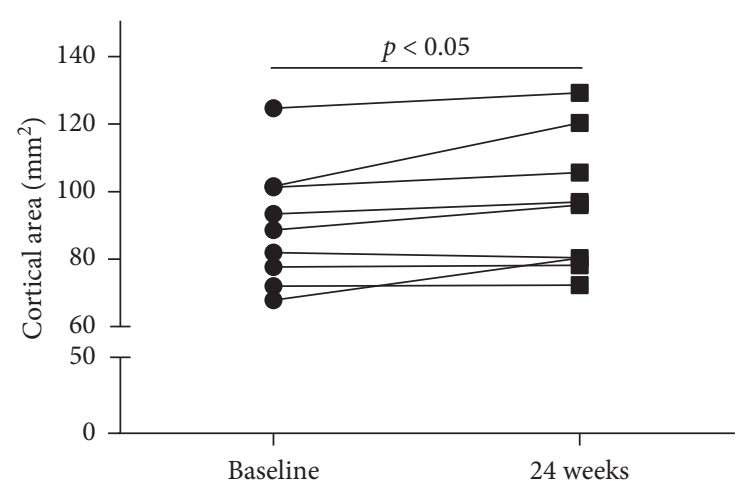

(c)

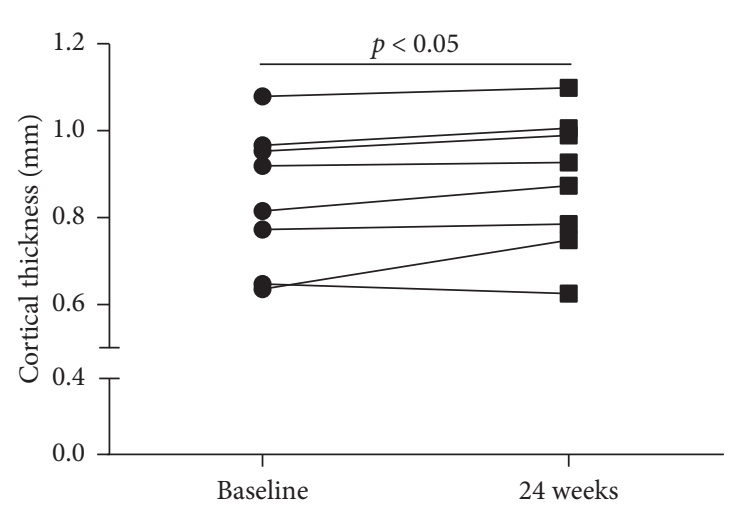

(e)

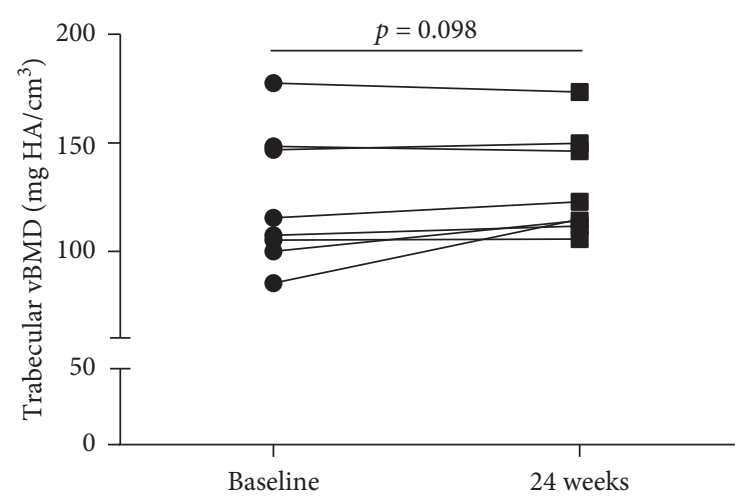

(g)

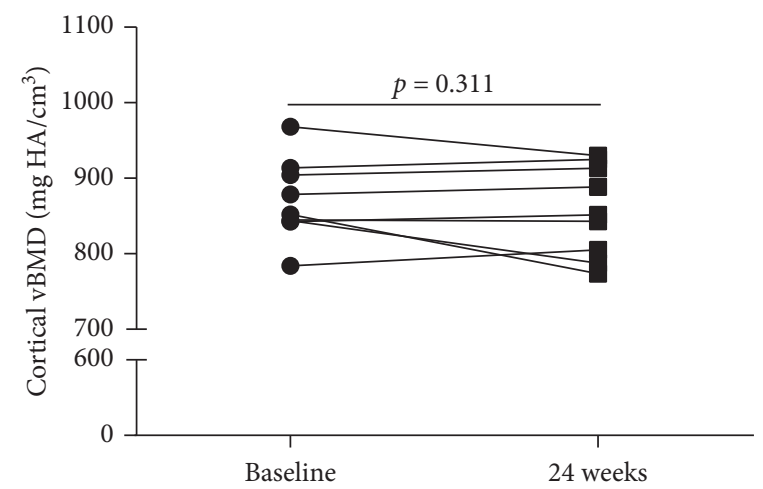

(b)

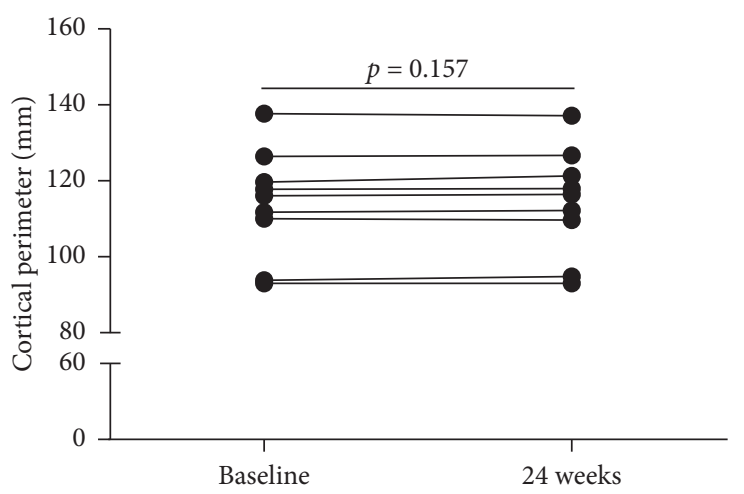

(d)

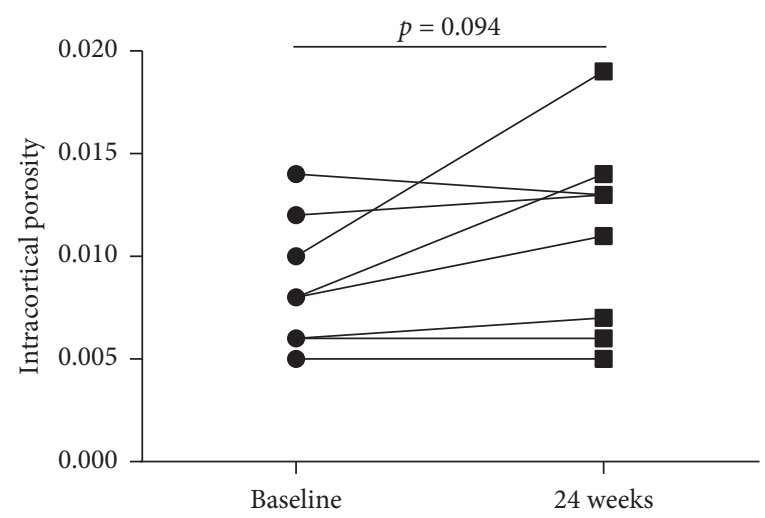

(f)

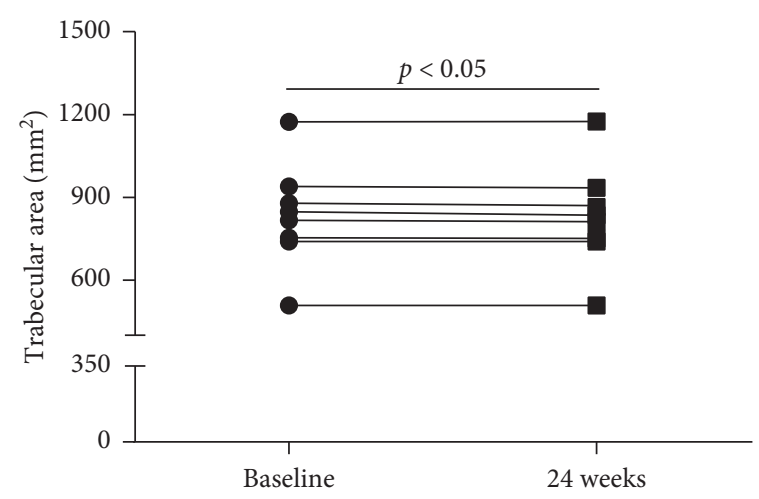

(h)

Figure 1: HR-pQCT parameters of the distal tibia at baseline and after 24 weeks of rhGH treatment of the patients. 
TABLE 4: Baseline and follow-up HR-pQCT parameters of the distal radius.

\begin{tabular}{|c|c|c|c|c|}
\hline & Baseline & 24-week follow-up & Changes & $p$ value \\
\hline Total vBMD $\left(\mathrm{mg} \mathrm{HA} / \mathrm{cm}^{3}\right)$ & $207.0 \pm 68.0$ & $208.1 \pm 69.1$ & $1.04 \pm 12.08$ & 0.802 \\
\hline Cortical area $\left(\mathrm{mm}^{2}\right)$ & $44.2 \pm 8.4$ & $46.5 \pm 9.7$ & $2.37 \pm 4.85$ & 0.181 \\
\hline Cortical vBMD (mg HA/ $\left.\mathrm{cm}^{3}\right)$ & $764.2 \pm 79.3$ & $751.5 \pm 73.5$ & $-12.66 \pm 26.73$ & 0.193 \\
\hline Cortical perimeter $(\mathrm{mm})$ & $74.1 \pm 10.0$ & $75.0 \pm 10.9$ & $0.50(-0.30,4.70)$ & 0.034 \\
\hline Cortical thickness (mm) & $0.602(0.495,1.187)$ & $0.599(0.500,1.209)$ & $0.034 \pm 0.089$ & 0.282 \\
\hline Intracortical porosity & $0.004 \pm 0.002$ & $0.006 \pm 0.004$ & $0.002 \pm 0.004$ & 0.077 \\
\hline Trabecular area $\left(\mathrm{mm}^{2}\right)$ & $275.7 \pm 75.5$ & $273.5 \pm 76.1$ & $-2.13 \pm 4.77$ & 0.216 \\
\hline Trabecular vBMD $\left(\mathrm{mg} \mathrm{HA} / \mathrm{cm}^{3}\right)$ & $113.4 \pm 36.4$ & $110.2 \pm 39.7$ & $-3.20 \pm 6.21$ & 0.161 \\
\hline Trabecular thickness (mm) & $0.208 \pm 0.013$ & $0.212 \pm 0.013$ & $0.004 \pm 0.003$ & 0.008 \\
\hline Trabecular number $(1 / \mathrm{mm})$ & $1.363 \pm 0.294$ & $1.291 \pm 0.325$ & $-0.071 \pm 0.078$ & 0.025 \\
\hline Trabecular separation (mm) & $0.743 \pm 0.175$ & $0.796 \pm 0.199$ & $0.053 \pm 0.054$ & 0.019 \\
\hline Tb.1/N.SD (mm) & $0.292 \pm 0.087$ & $0.317 \pm 0.096$ & $0.024 \pm 0.027$ & 0.026 \\
\hline Trabecular bone volume fraction & $0.168 \pm 0.046$ & $0.164 \pm 0.050$ & $-0.004 \pm 0.007$ & 0.134 \\
\hline
\end{tabular}

Data are presented as mean \pm SD or median and ranges. vBMD, volumetric bone mineral density; Tb.1/N.SD, Std. dev. of 1/Tb.N, inhomogeneity of network.

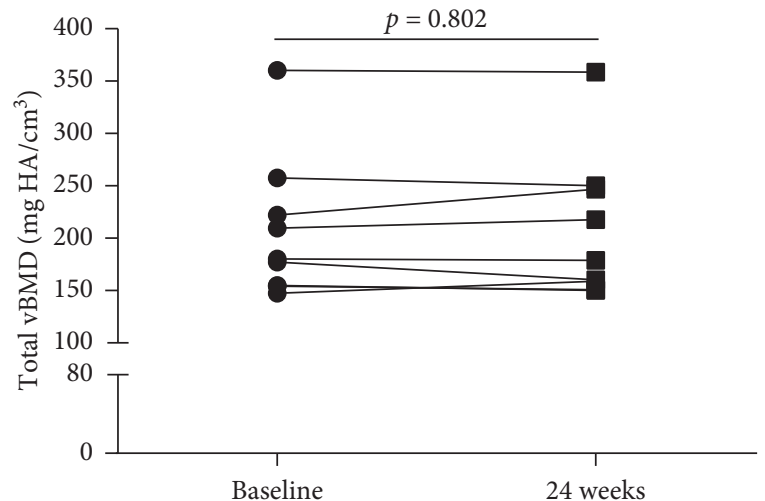

(a)

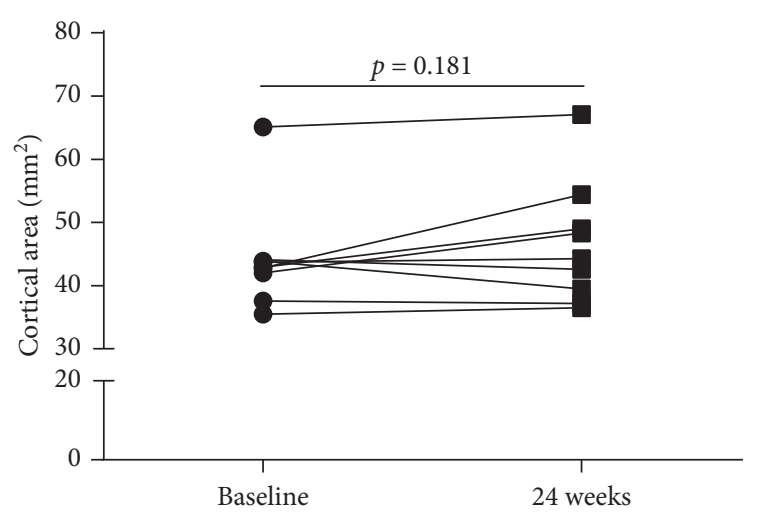

(c)

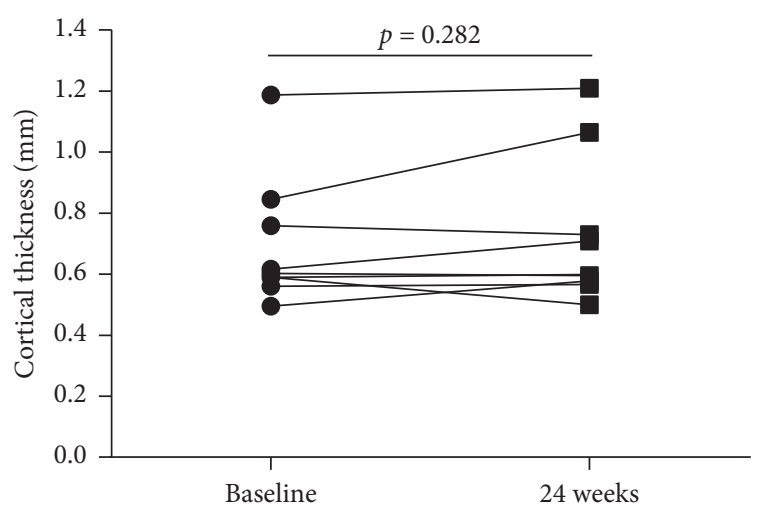

(e)

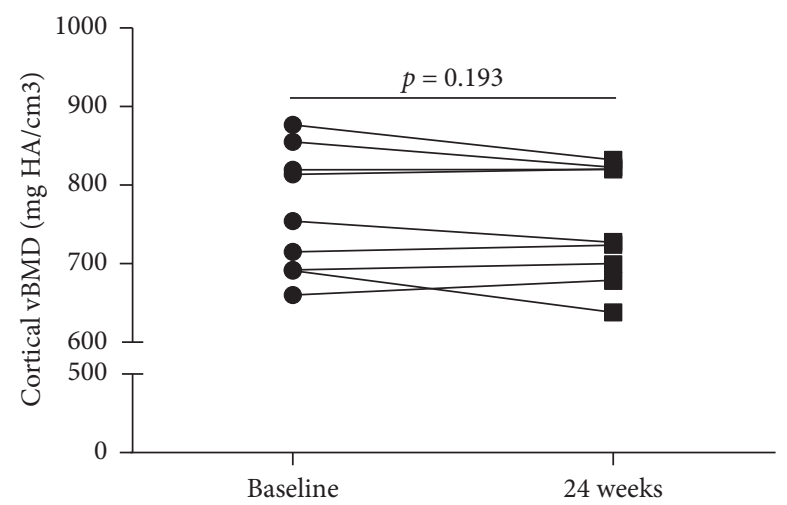

(b)

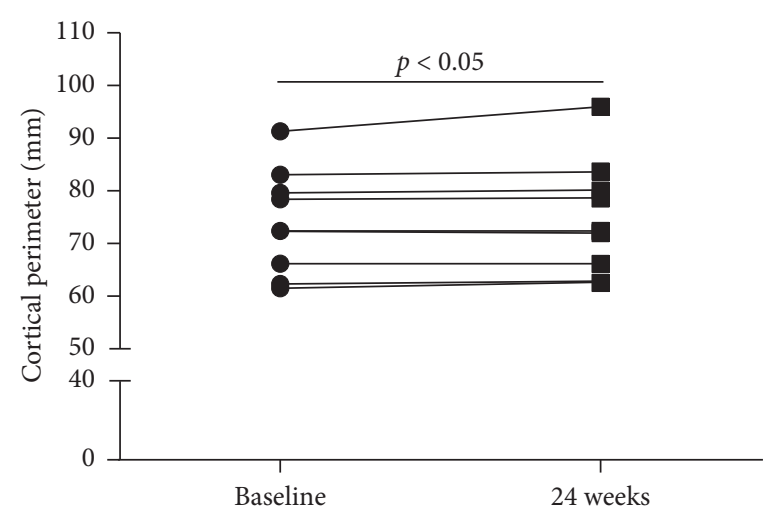

(d)

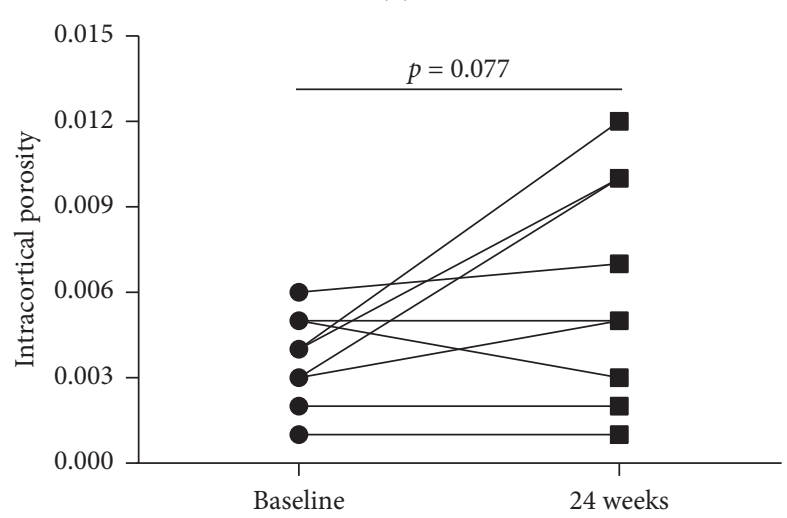

(f)

Figure 2: Continued. 


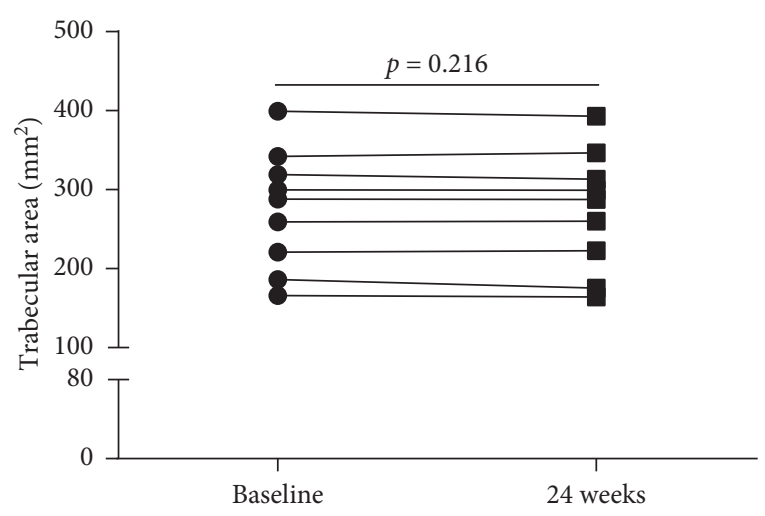

(g)

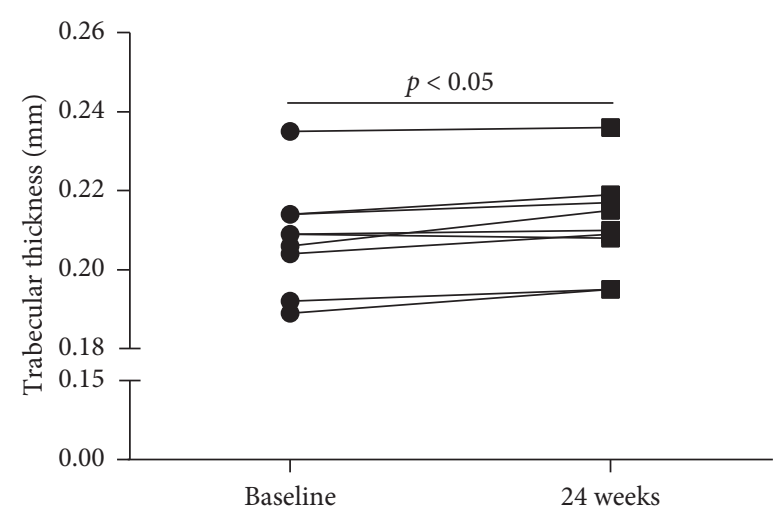

(h)

FIGURE 2: HR-pQCT parameters of the distal radius at baseline and after 24 weeks of rhGH treatment of the patients.

radius, accompanied by increased bone resorption biomarkers in a very early stage of treatment.

$\mathrm{GH} / \mathrm{IGF}-1$ axis is a pivotal regulator of bone homeostasis and is central to the achievement of normal longitudinal bone growth and bone mass [21]. GH and IGF-1 act in an endocrine, paracrine, or autocrine fashion to regulate osteoblast, osteocyte, and osteoclast function and thus sustains normal cortical and trabecular bone properties [21, 22]. Kinetic studies demonstrated that bone calcium deposition reaches a maximum during puberty, which is approximately five times that of adulthood [23]. Thus, loss of interactions of IGF-1 and gonadosteroids and parathyroid hormone is one of the main causes of impaired peak bone mass in adults with CO AGHD [23]. Although the effect of GH and IGF-1 on bone metabolism is widely accepted, pQCT microarchitecture results in AGHD patients are inconsistent. Bone microarchitecture was reported to be marginally reduced [24] or not changed [13] in some AGHD patients. In our previous work, CO AGHD patients had significantly decreased total vBMD, cortical vBMD, trabecular vBMD, cortical area, cortical thickness, trabecular thickness, and trabecular bone volume fraction of both tibia and radius [14].

Effects of rhGH on bone mineral density in patients with AGHD have been investigated in recent decades. In both patients with $\mathrm{CO}$ AGHD and AO AGHD, 18 months to 5 years of rhGH replacement therapy was reported to have various beneficial effects on BMD and BMC [8-11, 25]. There were also some studies which reported that short-term rhGH treatment increased bone turnover but did not increase BMC [26]. Evaluation of BMD and BMC in all these studies is based on DXA, which does not provide details of microarchitecture of cortical and cancellous bones and could not detect subtle changes of bone in early stages of rhGH treatment. HR-pQCT thus warrants shorter-term treatment for evidences of beneficial effects of rhGH in AGHD patients with low bone mass. Our data found that rhGH is beneficial for cortical bone, including increased cortical area, cortical perimeter, and cortical thickness. These data suggested that rhGH could promote subperiosteal bone formation. At the same time, the reduction of cancellous bone area suggested cortification of cancellous bone after rhGH treatment, which is significant for maintaining the mechanical stability of bone and increasing the antifracture ability of long bone after rhGH treatment. In consistent with reports from other groups, bone-resorption biomarker $\beta$-CTX was increased significantly after $\mathrm{rhGH}$ treatment in our study [7-9]. The baseline serum vitamin D level was insufficient in our patients, and vitamin D was not supplemented during this short-term pilot study in order to avoid confounding variables to evaluate the effect of rhGH on bone volumetric density and microstructure. Further studies are required to assess the add-on effect of vitamin $D$ in these patients.

The main limitation of this study is the small number of participants. But, there was good homogeneity in the etiology since all patients had CO AGHD due to MPHD, which is a distinct entity in AGHD and has different clinical patterns from AO AGHD. Another limitation is that there could be a selection bias in the study. Eight of the nine patients in the study undertook treatment with rhGH during childhood while the older one (51 years) never undertook treatment before enrollment. We enrolled this patient since this is a self-controlled prospective study. Another limitation is the relatively short term of treatment in our patients. We are going to follow-up these patients in long term and assess the dynamic changes of bone microarchitecture and risk of fracture in the future.

In conclusion, our findings suggest that 24 weeks of rhGH replacement induced a significant improvement in bone resorption markers and parameters of bone microarchitecture in male patients with childhood-onset adult growth hormone deficiency. CO AGHD should be recognized as a unique entity in AGHD and seamless transition management is advocated again.

\section{Data Availability}

The data used to support the findings of this study are available from the corresponding author upon request.

\section{Conflicts of Interest}

The authors declare that they have no conflicts of interest. 


\section{Authors' Contributions}

Hongbo Yang, Kemin Yan, and Yuping Xu contributed equally to this work. HY designed the study and wrote the primary manuscript. HZ and HP designed and supervised the study and revised the primary manuscript. KY took part in the collection of clinical data and analyzed the data. YX collected the pQCT data and WX supervised the data analysis of pQCT. QZ performed the biochemical measurements. LW contributed to the study management. FG helped to revise the primary manuscript.

\section{Acknowledgments}

This work was supported by the National Natural Science Foundation of China (No. 81970678) and the nonprofit Central Research Institute Fund of Chinese Academy of Medical Sciences (Nos. 2017PT32020 and 2018PT32001).

\section{References}

[1] M. E. Molitch, D. R. Clemmons, S. Malozowski, G. R. Merriam, and M. L. Vance, "Evaluation and treatment of adult growth hormone deficiency: an endocrine society clinical practice guideline," The Journal of Clinical Endocrinology \& Metabolism, vol. 96, no. 6, pp. 1587-1609, 2011.

[2] H. Yang, L. Wang, X. Qiu et al., "Body composition and metabolic health of young male adults with childhood-onset multiple pituitary hormone deficiency after cessation of growth hormone treatment," Journal of Pediatric Endocrinology and Metabolism, vol. 31, no. 5, pp. 533-537, 2018.

[3] S. J. Holmes, R. W. Whitehouse, J. E. Adams, and S. M. Shalet, "Reduced bone mineral density in patients with adult onset growth hormone deficiency," Journal of Clinical Endocrinology \& Metabolism, vol. 78, no. 3, pp. 669-674, 1994.

[4] T. Rosen, L. Wilhelmsen, G. Lappas, B. A. Bengtsson, and K. Landin-Wilhelmsen, "Increased fracture frequency in adult patients with hypopituitarism and GH deficiency," European Journal of Endocrinology, vol. 137, pp. 240-245, 1997.

[5] H. Holmer, J. Svensson, L. Rylander et al., "Fracture incidence in GH-deficient patients on complete hormone replacement including GH," Journal of Bone and Mineral Research, vol. 22, no. 12, pp. 1842-1850, 2007.

[6] M. S. Z. Berberoglu, F. Darendeliler, S. Poyrazoglu et al., "Evaluation of permanent growth hormone deficiency (GHD) in young adults with childhood onset GHD: a multicenter study," Journal of Clinincal Research in Pediatric Endocrinology, vol. 1, pp. 30-37, 2008.

[7] A. M. Ramos-Leví and M. Marazuela, "Treatment of adult growth hormone deficiency with human recombinant growth hormone: an update on current evidence and critical review of advantages and pitfalls," Endocrine, vol. 60, no. 2, pp. 203-218, 2018.

[8] G. Johannsson, T. Rosen, I. Bosaeus, L. Sjostrom, and B. A. Bengtsson, "Two years of growth hormone (GH) treatment increases bone mineral content and density in hypopituitary patients with adult-onset GH deficiency," Journal of Clinical Endocrinology \& Metabolism, vol. 81, no. 8, pp. 2865-2873, 1996.

[9] H. B. A. Baum, B. M. Biller, J. S. Finkelstein et al., "Effects of physiologic growth hormone therapy on bone density and body composition in patients with adult-onset growth hormone deficiency," Annals of Internal Medicine, vol. 125, no. 11, pp. 883-890, 1996.

[10] M. Bex, R. Abs, D. Maiter, A. Beckers, G. Lamberigts, and R. Bouillon, "The effects of growth hormone replacement therapy on bone metabolism in adult-onset growth hormone deficiency: a 2-year open randomized controlled multicenter trial," Journal of Bone and Mineral Research, vol. 17, no. 6, pp. 1081-1094, 2002.

[11] N. Bravenboer, P. J. Holzmann, J. C. ter Maaten, L. M. Stuurman, J. C. Roos, and P. Lips, "Effect of long-term growth hormone treatment on bone mass and bone metabolism in growth hormone-deficient men," Journal of Bone and Mineral Research, vol. 20, no. 10, pp. 1778-1784, 2005.

[12] P. Geusens, R. Chapurlat, G. Schett et al., "High-resolution in vivo imaging of bone and joints: a window to microarchitecture," Nature Reviews Rheumatology, vol. 10, no. 5, pp. 304-313, 2014.

[13] P. P. B. Silva, F. G. Amlashi, E. W. Yu et al., "Bone microarchitecture and estimated bone strength in men with active acromegaly," European Journal of Endocrinology, vol. 177, no. 5, pp. 409-420, 2017.

[14] H. Yang, K. Yan, X. Yuping et al., "Bone microarchitecture and volumetric bone density impairment in young male adults with childhood-onset growth hormone deficiency," European Journal of Endocrinology, vol. 180, no. 180, pp. 145-153, 2019.

[15] J. D. Wilson, "Peking Union Medical College Hospital, a palace of endocrine treasures," The Journal of Clinical Endocrinology \& Metabolism, vol. 76, no. 4, pp. 815-816, 1993.

[16] G. Mazziotti, S. Chiavistelli, and A. Giustina, "Pituitary diseases and bone," Endocrinology and Metabolism Clinics of North America, vol. 44, no. 1, pp. 171-180, 2015.

[17] T. B. VanItallie, M. U. Yang, S. B. Heymsfield, R. C. Funk, and R. A. Boileau, "Height-normalized indices of the body's fatfree mass and fat mass: potentially useful indicators of nutritional status," The American Journal of Clinical Nutrition, vol. 52, no. 6, pp. 953-959, 1990.

[18] J. A. MacNeil and S. K. Boyd, "Improved reproducibility of high-resolution peripheral quantitative computed tomography for measurement of bone quality," Medical Engineering \& Physics, vol. 30, no. 6, pp. 792-799, 2008.

[19] Y. Pauchard, A.-M. Liphardt, H. M. Macdonald, D. A. Hanley, and S. K. Boyd, "Quality control for bone quality parameters affected by subject motion in high-resolution peripheral quantitative computed tomography," Bone, vol. 50, no. 6, pp. 1304-1310, 2012.

[20] S. Boutroy, M. L. Bouxsein, F. Munoz, and P. D. Delmas, "In vivo assessment of trabecular bone microarchitecture by highresolution peripheral quantitative computed tomography," Journal of Clinical Endocrinology and Metabolism, vol. 90, no. 12, pp. 6508-6515, 2005.

[21] A. Giustina, G. Mazziotti, and E. Canalis, "Growth hormone, insulin-like growth factors, and the skeleton," Endocrine Reviews, vol. 29, no. 5, pp. 535-559, 2008.

[22] S. Yakar, H. Werner, and C. J. Rosen, "Insulin-like growth factors: actions on the skeleton," Journal of Molecular Endocrinology, vol. 61, pp. T115-T137, 2018.

[23] S. A. Abrams, "Calcium turnover and nutrition through the life cycle," Proceedings of the Nutrition Society, vol. 60, pp. 283-289, 2001.

[24] R. D. Murray, J. E. Adams, and S. M. Shalet, "A densitometric and morphometric analysis of the skeleton in adults with varying degrees of growth hormone deficiency," The Journal 
of Clinical Endocrinology \& Metabolism, vol. 91, no. 2, pp. 432-438, 2006.

[25] G. S. Conway, M. Szarras-Czapnik, K. Racz et al., "Treatment for 24 months with recombinant human GH has a beneficial effect on bone mineral density in young adults with childhood-onset GH deficiency," European Journal of Endocrinology, vol. 160, no. 6, pp. 899-907, 2009.

[26] S. A. Beshyah, E. Thomas, P. Kyd et al., "The effect of growth hormone replacement therapy in hypopituitary adults on calcium and bone metabolism," Clinical Endocrinology, vol. 40, pp. 383-391, 1994. 